\title{
Latest experimental advances in nonlinear multimode fiber optics
}

\author{
Y. Leventoux ${ }^{1}$, M. Fabert ${ }^{1}$, M. Săpânțan ${ }^{1}$, K. Krupa ${ }^{2}$, A. Tonello ${ }^{1}$, G. Granger ${ }^{1}$, S. Février ${ }^{1}$, \\ T. Mansuryan ${ }^{1}$, A. Niang ${ }^{3}$, B. Wetzel ${ }^{1}$, G. Millot ${ }^{4,5}$, S. Wabnitz ${ }^{6,7}$, and V. Coudere ${ }^{1}$ \\ 1. Université de Limoges, XLIM, UMR CNRS 7252, 123 Avenue A. Thomas, 87060 Limoges, France \\ 2. Institute of Physical Chemistry Polish Academy of Sciences, ul. Kasprzaka 44/52, 01-224 Warsaw, Poland \\ 3. Dipartimento di Ingegneria dell'Informazione, Università degli Studi di Brescia, via Branze 38, 25123, Brescia, Italy \\ 4. Université Bourgogne Franche-Comté, ICB UMR CNRS 6303, 9 Avenue A. Savary, 21078 Dijon, France \\ 5. Institut Universitaire de France (IUF), 1 rue Descartes, Paris, France \\ 6. DIET, Sapienza Università di Roma, Via Eudossiana 18, 00184 Roma, Italy \\ 7. Novosibirsk state University, Ulitsa Pirogova, 2, Novosibirskaya oblast'630090, Russia
}

Over the last few years, we have experienced a renaissance of nonlinear optics in multimode fibers, driven by both fundamental and applied research. Multimode optical fibers (MMFs) offer a natural environment for investigating multidimensional systems and their complex collective dynamics. Indeed, numerous new nonlinear effects have recently been observed including, for instance, Kerr beam self-cleaning, mode-locking by spatiotemporal filtering, or multimode parametric wave mixing, to name a few [1]. On the other hand, from the application perspective, MMFs are expected to provide interesting novel solutions for high power delivery and increased transmission capacity. Significant work is now in progress to exploit the recently discovered dynamics, and improve the performances of different technologies such as laser endoscopy, nonlinear imaging, and fiberbased laser sources [2,3]. In addition, the recent development of advanced measurement techniques, permitting to capture the full spatiotemporal nature of the multimode phenomena has also attracted a strong research interest [4].
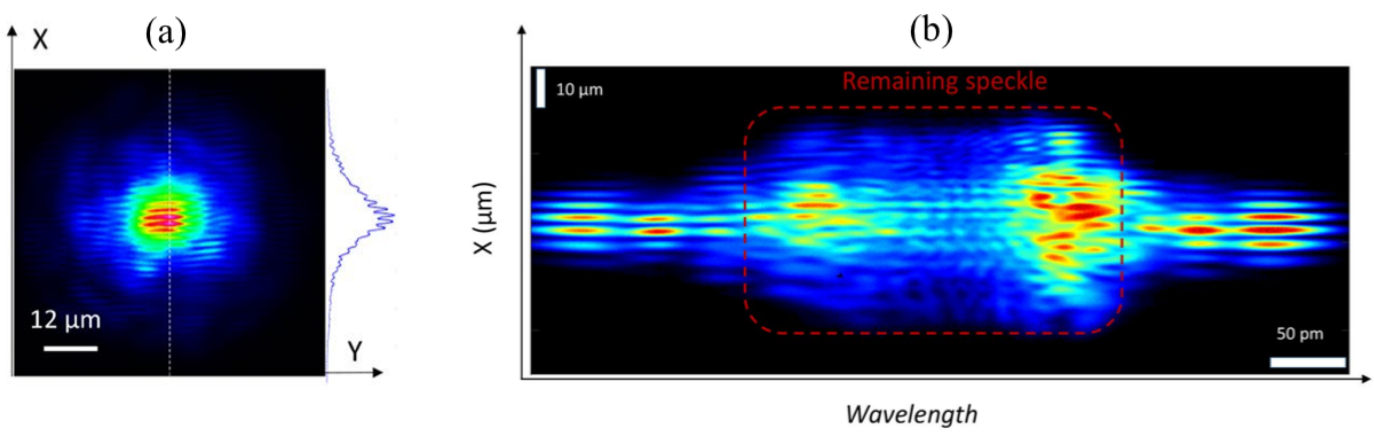

Fig. 1 Experimental multimode phase-locking. (a) Spatial interference pattern obtained from two independently self-cleaned beams. (b) Same interference pattern but spectrally decomposed along wavelength. Vertical axis corresponds to $\mathrm{x}$ transverse dimension.

In this talk, we will review some of the most recent advances of nonlinear pulse propagation in multimode optical fibers. We will present experimental results that may open new perspectives for coherent and scalable multimode beam combining. Specifically, we demonstrated that the Kerr-induced self-cleaning mechanism preserves the beams' mutual coherence via a noise-free parametric process. Indeed, we have shown that it is possible to generate two independently self-cleaned beams with a mutual coherence high enough to produce a clear stable fringe pattern at the output of a nonlinear interferometer [5]. Next, we will discuss the possibility to induce single-mode short-wave/mid-infrared supercontinuum based on the recently observed space-time effect, the so-called geometric parametric instability in graded-index MMFs. Finally, we will present a new 3D mapping technique [6] that we developed in two versions: (1) providing a time-resolved beam image with picosecond resolution, and (2) allowing for spectrally-resolved spatial measurements with sub-nanometer resolution.

\section{References}

[1] K. Krupa, A. Tonello, A. Barthélémy, T. Mansuryan, V. Couderc, G. Millot, Ph. Grelu, D. Modotto, S. Babin and S. Wabnitz, "Multimode nonlinear fiber optics, a spatiotemporal avenue," APL Photonics 4, 110901 (2019).

[2] W. Fu, L. G. Wright, P. Sidorenko, S. Backus, and F. W. Wise, "Several new directions for ultrafast fiber lasers," Opt. Express 26, 9432-9463 (2018).

[3] Y. Zhang, Y. Cao, J.-X. Cheng, "High-resolution photoacoustic endoscope through beam self-cleaning in a graded index fiber," Opt. Lett. 44, 3841-3844 (2019).

[4] J. C. Jing, X. Wei, and L. V. Wang, "Spatio-temporal-spectral imaging of non-repeatable dissipative soliton dynamics," Nat. Comm. 11, $2059(2020)$.

[5] M. Fabert, M Săpânțan, K. Krupa, A. Tonello, Y. Leventoux, S. Février, T. Mansuryan, A. Niang, B. Wetzel, G. Millot, S. Wabnitz, Vincent Couderc," Coherent combining of self-cleaned multimode beams," Sci. Rep. 10, 20481 (2020).

[6] Y. Leventoux, G. Granger, K. Krupa, A. Tonello, G. Millot, M. Ferraro, F. Mangini, M. Zitelli, S. Wabnitz, S. Février, V. Couderc, "3D time-domain beam mapping for studying nonlinear dynamics in multimode optical fibers," Opt. Let. 46, 66 (2021). 